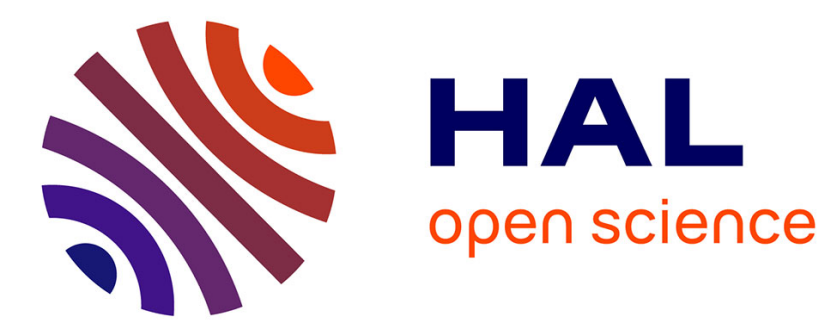

\title{
Theoretical study of the interaction between carbon nanotubes and carboplatin anticancer molecules
}

Mohamed El Khalifi, Eric Duverger, Hatem Boulahdour, Fabien Picaud

\section{To cite this version:}

Mohamed El Khalifi, Eric Duverger, Hatem Boulahdour, Fabien Picaud. Theoretical study of the interaction between carbon nanotubes and carboplatin anticancer molecules. Analytical Methods, 2015, 7 (24), pp.10145 - 10150. hal-02868341

\section{HAL Id: hal-02868341 https://hal.science/hal-02868341}

Submitted on 15 Jun 2020

HAL is a multi-disciplinary open access archive for the deposit and dissemination of scientific research documents, whether they are published or not. The documents may come from teaching and research institutions in France or abroad, or from public or private research centers.
L'archive ouverte pluridisciplinaire HAL, est destinée au dépôt et à la diffusion de documents scientifiques de niveau recherche, publiés ou non, émanant des établissements d'enseignement et de recherche français ou étrangers, des laboratoires publics ou privés. 


\section{Analytical Methods}

\section{Accepted Manuscript}

\section{Analytical Methods}
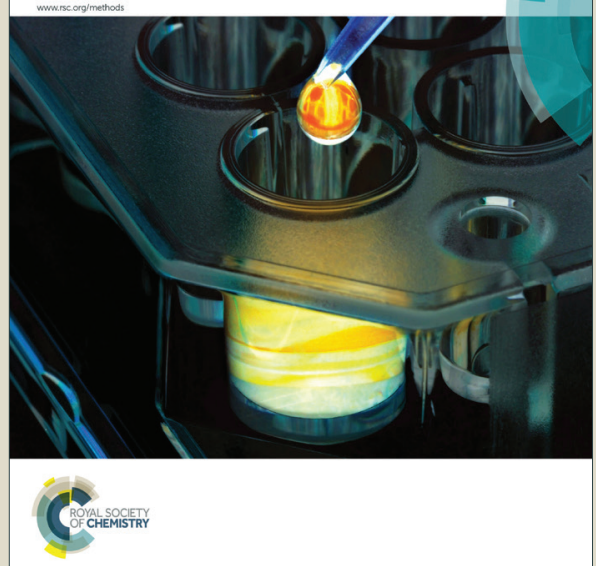

This is an Accepted Manuscript, which has been through the Royal Society of Chemistry peer review process and has been accepted for publication.

Accepted Manuscripts are published online shortly after acceptance, before technical editing, formatting and proof reading. Using this free service, authors can make their results available to the community, in citable form, before we publish the edited article. We will replace this Accepted Manuscript with the edited and formatted Advance Article as soon as it is available.

You can find more information about Accepted Manuscripts in the Information for Authors.

Please note that technical editing may introduce minor changes to the text and/or graphics, which may alter content. The journal's standard Terms \& Conditions and the Ethical guidelines still apply. In no event shall the Royal Society of Chemistry be held responsible for any errors or omissions in this Accepted Manuscript or any consequences arising from the use of any information it contains. 


\title{
Theoretical study of the interaction between carbon nanotubes and carboplatin anticancer molecules
}

\author{
M. El Khalifi ${ }^{\mathrm{a}}$, E. Duverger ${ }^{\mathrm{b}}$, H. Boulahdour ${ }^{\mathrm{a}}$ and F. Picaud ${ }^{\mathrm{a},{ }^{*}}$. \\ Full DFT calculations were investigated to study the interactions between single wall functionalized carbon-based metallic \\ nanotubes (CNTs) with carboplatin anticancer drugs (CPT). The geometry of the CNT-carboplatin was optimized considering \\ different molecular configurations on inner and outer surfaces of the nanotube. Simulation results show that the most stable \\ physisorption state for molecules is located inside the nanotube in a parallel configuration. More, we demonstrated that the \\ molecular physisorption was reinforced as soon as the number of encapsulated carboplatin molecules increased, leading to a \\ favored state where the nanovector is fulfilled by the drug. Moreover, all theoretical results show that the therapeutic agent is not \\ affected when it is attached onto CNTs.
}

Keywords: carbon nanotubes; therapeutic agents; DFT calculations

\section{A. Introduction}

Although fields to develop efficient drug delivery systems extend into all therapeutics classes of pharmaceuticals, many of them suffer because of their limited ability to reach the target tissue. Moreover, the serious adverse effects observed when chemotherapeutic agents are systemically administered confirmed their poor specificity in reaching tumor tissues ${ }^{1}$. More, the efficacy of many potent and promising drug molecules is limited by their low water solubility, by the increased drug resistance and by their highly cytotoxic side effects ${ }^{2}$. To circumvent such important drawbacks, an efficient way of systemic transportation needs to be developed. Many of the pharmacological properties of conventional drugs can be improved through the use of nanocarriers ${ }^{3}$ incorporating chemotherapeutics drug and specific cancer cells receptors, despite some inconvenient ${ }^{4-7}$. Ideally, this class of novel drug carriers must fulfill the following three criteria: efficient encapsulation of the drug, transport of this drug to the targeted region, and successful drug release at the specific cancer cell location. In recent years, various types of drug/agent nanocarriers were investigated but few were commercialized or used in clinical studies ${ }^{8}$. Practically, loading of small molecules such as chemotherapeutic cancer drugs can proceeds following two main strategies, i.e. covalent and non-covalent bindings. Both 
of them should follow the five Lipinski rules, crucial to determine whether a chemical compound has enough properties to become an active drug for humans 9 .

Among all drug carriers, carbon nanotubes (CNTs) have attracted a wide attention for biologically relevant molecules, because of their unique physicochemical and biological properties ${ }^{7,10-13}$

Many of the conventional anti-cancer drugs were loaded into $\mathrm{CNTs}^{14,15}$. For instance, paclitaxel ${ }^{16}$, doxorubicin ${ }^{15,17}$ and platinum complexes ${ }^{18,19}$ loaded onto CNTs have demonstrated their efficiency for in vitro and in vivo treatments. Apparently, the net advantage of the well-functionalized CNT concerns the diminution of toxicity. Indeed, unfunctionalized CNT aggregated in the lung can cause pulmonary toxicity and inflammation, while biocompatible functionalized CNT exhibits remarkably reduced in vivo toxicity after being intravenously injected into animal ${ }^{20-22}$. Non-covalent adsorption was used to incorporate multiple therapeutics, diagnostic, and targeting agents inside one nanocapsule ${ }^{23-26}$. This latter one appears then as a cargo designed to deliver the drug in the tumor. Nevertheless, the size and the cap of these nanocapsules are not easy to manufacture ${ }^{27}$ and many external factors such as $\mathrm{pH}$ and salt concentrations can affect this non-covalent bonding.

Recently we studied the interactions between CNTs and different molecules, such as azomethine $\left(\mathrm{C}_{2} \mathrm{H}_{5} \mathrm{~N}\right)$ and an anticancer agent (Pt(IV) complex) linked to an amino-derivative chain. Our results showed that the most stable chemisorption state for both molecules was located outside the nanotube corroborating the experimental observations of the Prato's group ${ }^{28}$. On CNT, the direct attack of the $(1,2)$ carbon bond was clearly preferential. Attaching longer amino derivatives and a subsequent $\mathrm{Pt}(\mathrm{IV})$ complex onto these carbon-based nanostructures do not change the results ${ }^{29}$.

At the light of these examples, it is thus crucial to correctly select and control the surface coating of the nanotemplates to achieve specific aims in CNT based biomedical applications. Many questions must be addressed when a drug is in interaction with a surface. The first ones concern the adsorption itself. What is the most stable position between the vector and the molecule envisioned? How does the adsorption influence the electronic distribution of valence charge? The second ones are more specific to the drug. One has to know whether the electronic structure, activity, and conformation of the drug are modified when it is in interaction with the carbon nanotube. In this paper, we use full density functional theory (DFT) calculations within the SIESTA approach to investigate these questions ${ }^{30}$. We report some new theoretical results concerning the adsorption of carboplatin molecule inside and outside CNTs. Thanks to full DFT calculations we determine the route to stabilize the cytotoxic anticancer drug carboplatin (CPT) inside and outside CNTs, and we demonstrate that carboplatin can find stable geometry leading to strong adsorption into $(10,10) \mathrm{CNT}$ corroborating the experimental observations of the Hampel's group ${ }^{31,32}$. Moreover, the close analysis of the electronic properties in terms of density of states (DOS), electronic charge distribution and charge transfer in the case of the CNT plus drug molecule shows a weak modification of the drug molecule properties when adsorbed on (or in) the nanotube. Then, when the most preferential adsorption was identified, the adsorption of a second carboplatin molecule was studied. We 
demonstrated that the increase of carboplatin molecule does not fundamentally perturb the platinum complex, and better stabilized it, leading to a most favourable stable state where the CNT was fulfilled by the therapeutic agent.

\section{B. Theoretical details}

We studied system consisting of an armchair single-walled $(10,10)$ CNT with tube diameter equal to $13.54 \AA$ (mean diameter found in experimental CNTs growth ${ }^{33,34}$ ) and containing 160 hexagon carbon rings. The length of the carbon tube in the slab was chosen to be equal to $19.7 \AA$ and the total number of carbon atoms forming the tube was thus equal to 340.40 Hydrogen atoms were added to saturate $\mathrm{C}$ dangling bonds at both tube ends in order to reproduce a correct experimental setup for opened tubes ${ }^{35,36}$ leading to a total length equal to $21.5 \AA$ as depicted in Figure 1. This allows to treat the possible oxidation of CNT when solvated and to avoid dangling bonds at their opened edge, that could dramatically modify their reactivity when the therapeutic try encapsulating. Carboplatin $\left(\mathrm{C}_{6} \mathrm{H}_{12} \mathrm{~N}_{2} \mathrm{O}_{4} \mathrm{Pt}\right)$ drug molecule was studied in gas phase with the same unit cell of the CNT tube (i.e. 40 x $40 \times 50) \AA^{3}$. The last number represents the length of the unit cell along the tube axis. Given the large unit cell, the Brillouin zone was sampled using a single k-point at the center $\square$. A basis set of localized atomic orbitals (double- $\square$ plus polarization functions), and norm-conserving pseudopotentials were employed. Since experimental proofs demonstrated the possible release of CPT from CNT for any $\mathrm{pH}$ values of the culture medium, we chose to perform simulations without any Van der Waals corrections $^{32}$.

a)

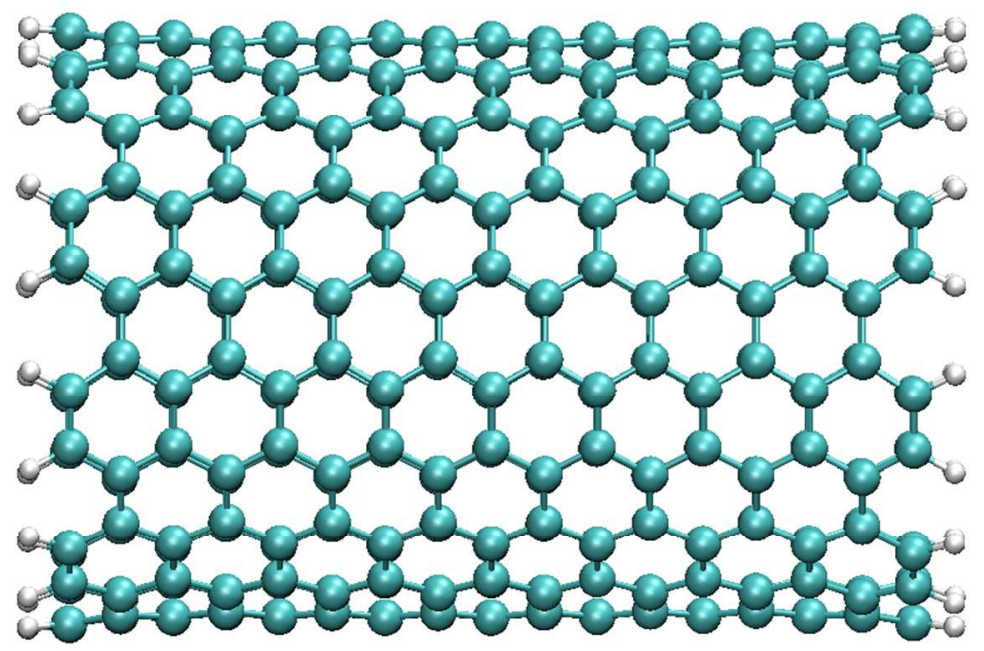


b)

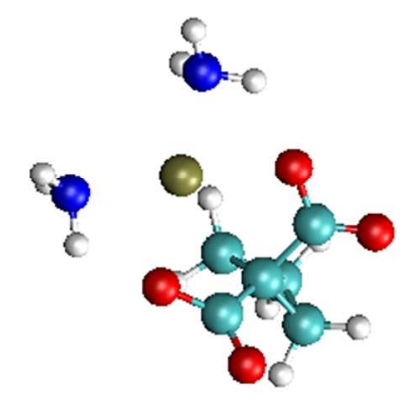

Figure 1: Optimized Geometry of (a) single-walled $(10,10) \mathrm{CNT}$ with hydrogen, (b) Carboplatin $\left(\mathrm{C}_{6} \mathrm{H}_{12} \mathrm{~N}_{2} \mathrm{O}_{4} \mathrm{Pt}\right)$.

The adsorption energy (Eads) of adsorbed molecules (Mol) on the inner (or outer) surface of the CNT (Template) was derived from the energy difference between the different states of the system, namely, $\mathrm{E}_{\mathrm{ads}}=\mathrm{E}(\mathrm{Mol}+$ Template) $-\mathrm{E}(\mathrm{Template})-\mathrm{E}(\mathrm{Mol})$. A negative $\mathrm{E}_{\text {ads }}$ value denoted a more favorable interaction between the drug and the CNT.

The total energies were obtained from ab initio calculations in the framework of Kohn-Sham realization of the density functional theory DFT ${ }^{37,38}$. We used the generalized gradient approximation (GGA) in the form proposed by Perdew et al. ${ }^{39}$ for the exchange correlation density functional as implemented in the SIESTA package ${ }^{30}$. All calculations were performed without spin polarization. We use the self-consistency mixing rate of 0.1 , a maximum force tolerance of $0.01 \mathrm{eV} / \mathrm{A}$ and a mesh cut off of 100 Ry (the variations of these parameters showed a very low perturbation of the total energies by less than $0.1 \%$ ). The selfconsistent cycles were stopped when variations of the total energy per unit cell and band structure energy were both less than $10^{-4}$ $\mathrm{eV}$. Bader charge analysis in order to evaluate the modification of valence charge on the molecule and the Pt atom were performed using the program developed by Henkelman et al. ${ }^{40-43}$.

\footnotetext{
C. Results

Carboplatin is a current chemotherapy drug used against several types of cancer. Since its introduction in the late 1980s, it became popular in clinical treatment due to its vastly reduced side-effects ${ }^{44}$. It corresponds to the second generation of platinum agent showing higher solubility in water and fewer side-effects than its parent agent (cisplatin) ${ }^{45}$.
}

a) Adsorption in outer surface 
To study the interaction between the drug molecule and its carrier, we first placed it on the outer surface of the CNT (Figure 2a). Indeed, this adsorption site will be always accessible for the molecule while the inner surface is linked to the pore aperture. The optimization of the molecule geometry shows that the molecule tends to lie near the surface with an energy minimum equal to $-0.13 \mathrm{eV}$. Calculated electronic density of states (DOS) for this optimized position is presented in Figure 2-b with the DOS of the molecule alone (Figure 2-c), as a function of the energy. We observe some discrepancies in the DOS height and slight shifts of energy levels of CPT due to interaction with CNTs. We retrieve this slight discrepancy in the sum of the entire valence electronic charge of the CPT molecule. In the gas phase configuration, the valence electronic charge is equal to $80.000 \mathrm{e}$ and to $80.007 \mathrm{e}$ near the CNT outer surface (variation of $0.007 \mathrm{e}$ ). It should be noted that the Pt valence charge is $9.53 \mathrm{e}$ in gas phase and $9.51 \mathrm{e}$ near the CNTs outer surface. The Bader's charges analysis shows that the overall electronic valence charge is kept. These data demonstrate that the CPT stabilization on the CNT surface maybe is possible but rather low to block the molecule on the carrier until its release.

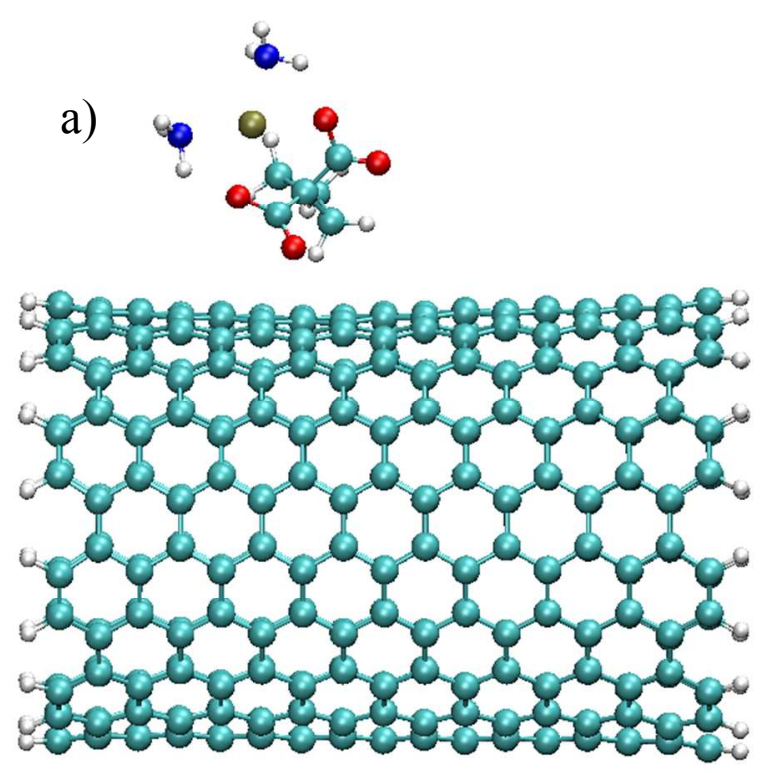



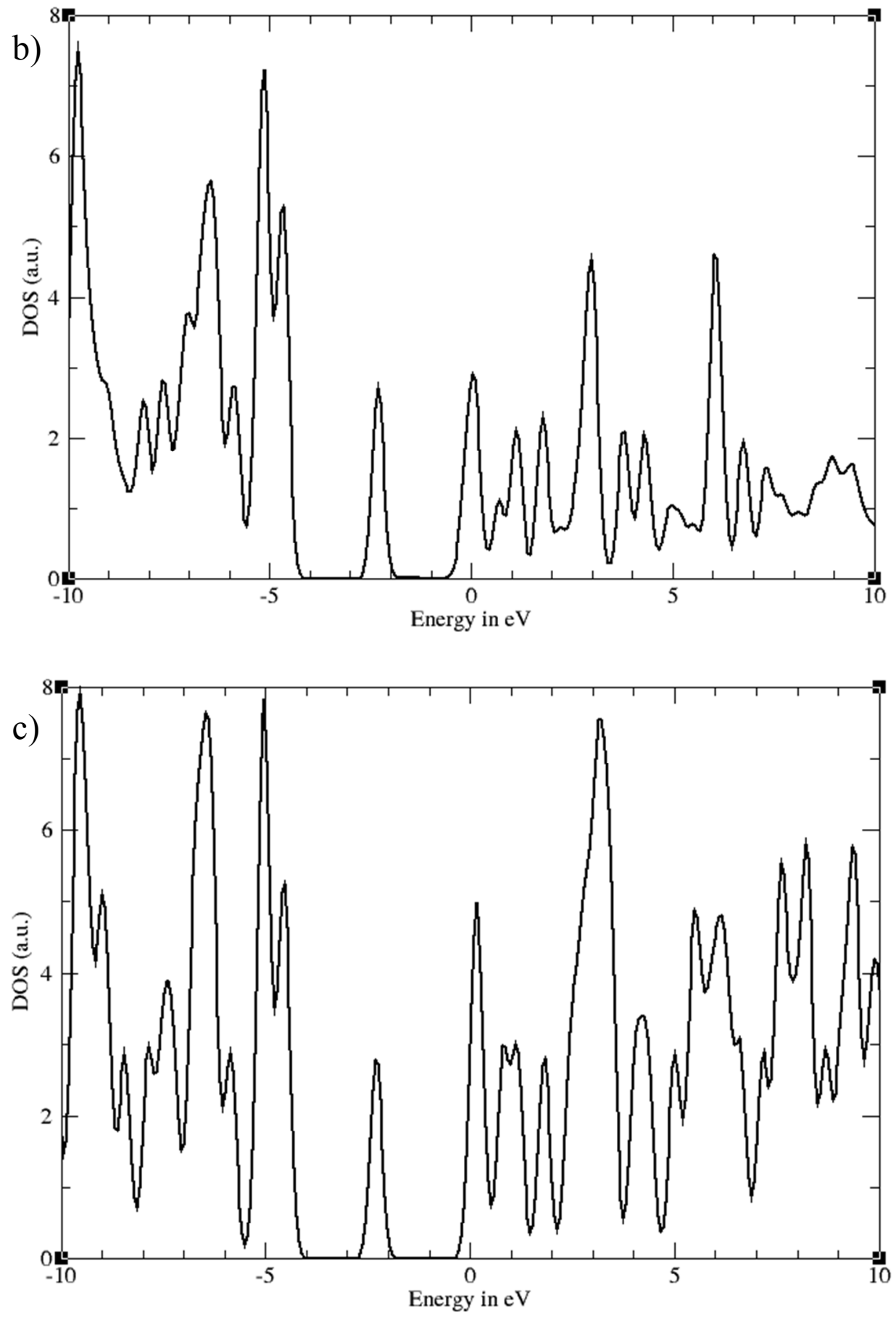

Figure 2: a) Geometry of the carboplatin-CNT final relaxed position when starting atop carbon hexagon. b) CPT DOS for the carboplatin-CNT presented Figure 2-a, c) CPT DOS in gas phase. 


\section{b) Drug encapsulation}

To investigate how the carboplatin molecule interacts with inner surface of CNTs, the molecule was placed in a symmetric position along its principal z-axis (Figure 3). The molecule was localized first at an arbitrary distance and its position was optimized. We deduced from this calculation the mean algebraic distance between the Pt atom of CPT and the CNT centre of mass, denoted as $d$. Then, we progressively changed the position of the molecule and calculated the new optimized value of $d$ to simulate the translation of the whole molecule from $21 \AA$ to $-21 \AA$ across the CNT. For each distance, the whole structure was relaxed by allowing atoms displacement to minimize the total energy.

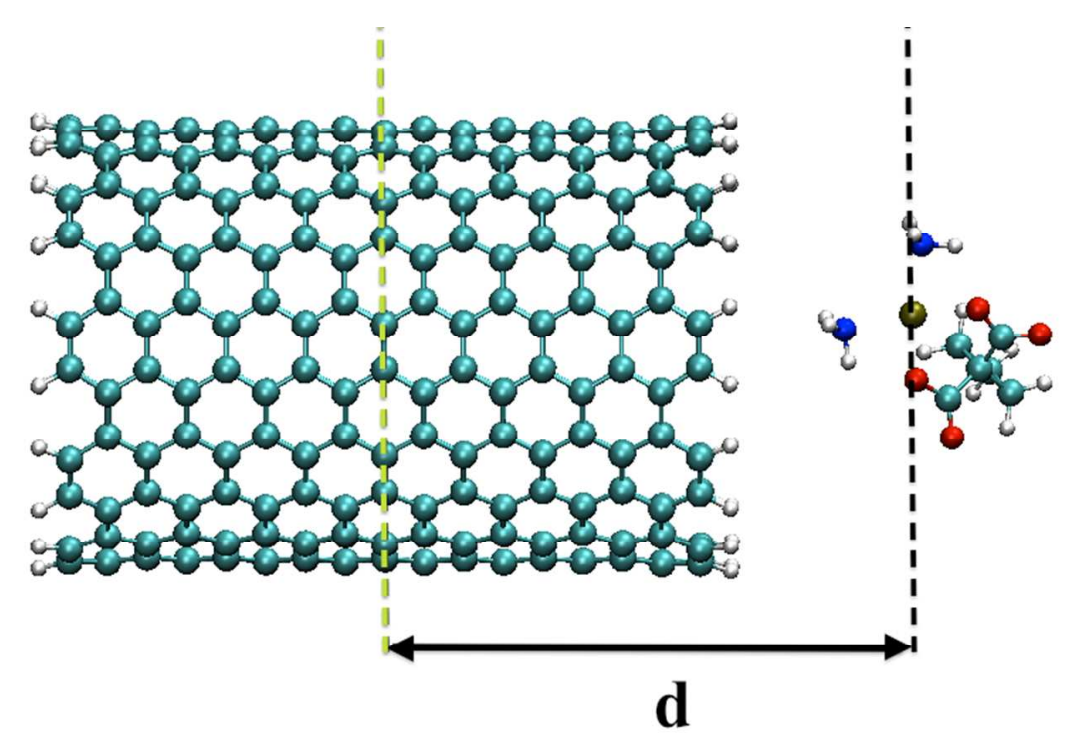

Figure 3: Geometry of the carboplatin-CNT starting configuration, the dashed green line represents the algebraic distance d between the $\mathrm{z}$-origin and the $\mathrm{Pt}$ atom $(\mathrm{d}>0$ at the right; $\mathrm{d}<0$ at the left).

The adsorption energy for the interacting system is presented in Figure 4. We can observe two small potential barriers of height around $0.07 \mathrm{eV}$ at about $10 \AA$ of the CNT edges (denoted (1)-(2)). As far as the molecule passes through these energy barriers, it feels a large attractive valley which decreases until $-0.55 \mathrm{eV}$ for $\mathrm{d}=-4.1 \AA$ (denoted (3)). This position corresponds to the most stable adsorption site for this study. For larger d (d ranging from $-2.0 \AA$ to $9.0 \AA$ ), another feeble potential barrier with a height equal to $0.07 \mathrm{eV}$ appears (denoted (4)-(5) on the Figure 4). We can note that the energy diffusion valley is slightly dissymmetric due to the asymmetry of the CPT. All these results show that the molecule can be confined inside the CNT. More, in this conformation, it should raise an energy barrier to exit from the nanocarrier equal to $0.55 \mathrm{eV}$, with a large spatial extension (around $10 \AA$ of the CNT carbon edges). This energy adsorption path permits to have a hint of the total diffusion path across the CNT. 


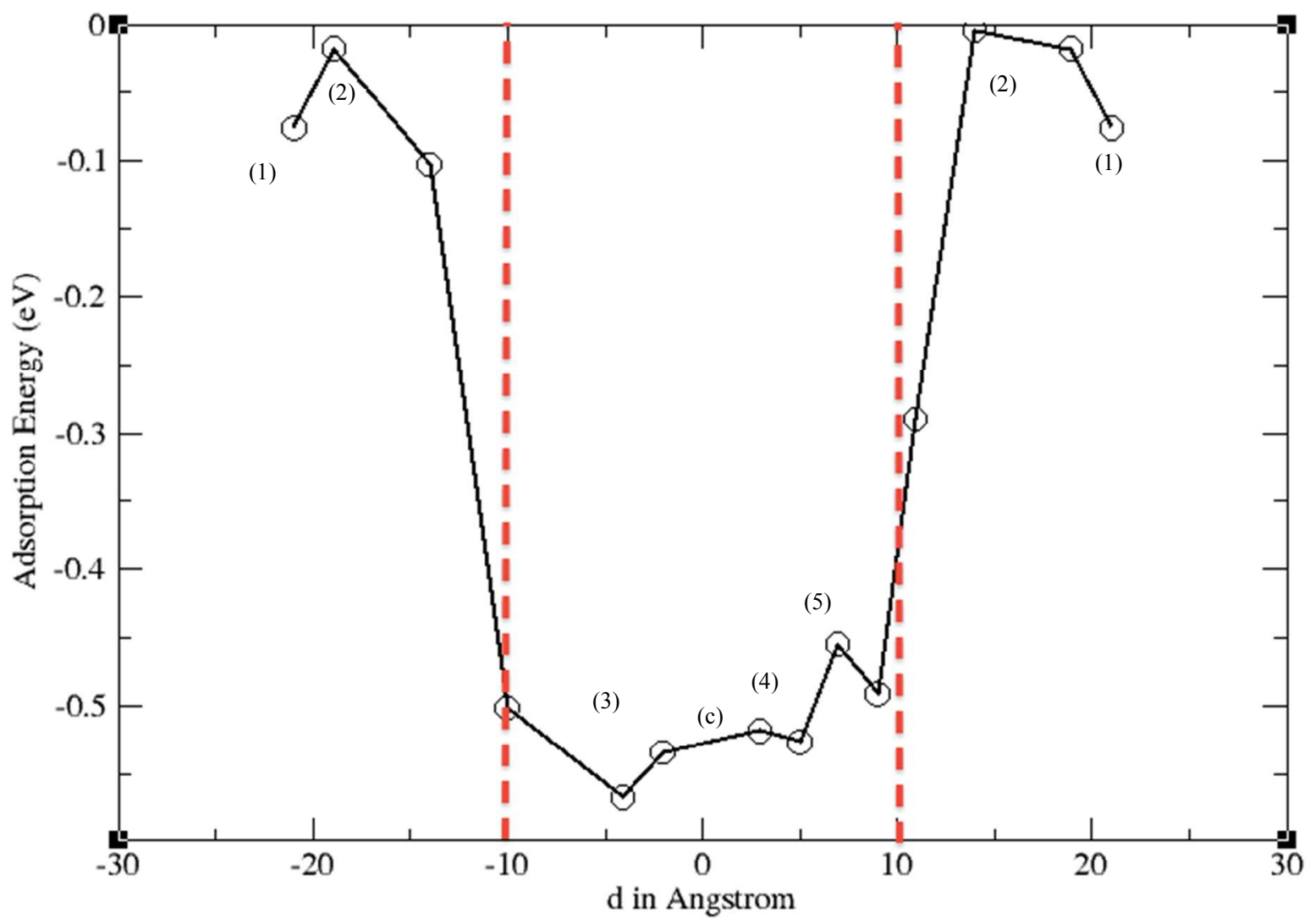

Figure 4: interaction energy of CPT as a function of $\mathrm{d}$ (defined as the distance between the absolute position of Pt atom in the CPT molecule and the CNT center of mass. Red bars highlight the CNT carbon edges.

The analyses of the Bader's charges were realized for the whole CPT molecule and, more particularly on the Pt atom different position depicted in Fig. 4 (positions (1), (3) and (c)). For the valence electronic charge in the position (1), we obtain 80.003 e with a valence charge centred onto Pt atom equal to 9.544 e. The valence charge of the CPT increases at 80.090 e in the position (3) and diminishes very slightly at 80.085 e for position (c). On the contrary, the valence charge centred onto Pt atom stays constant and equal to 9.524 e. All these data show that the overall electronic valence charge is conserved despite the presence of the CNT nanocarrier. In Figure 5, we present the calculated electronic density of states (DOS) for the CPT molecule in position (3) with the DOS of an isolated CPT gas molecule as a function of the energy. Despite some small discrepancies (height of DOS and shifts of energy levels) due to interaction with CNTs, the inert and non-reactive quality of CNTs is confirmed when the encapsulation of carboplatin took place. 
a)
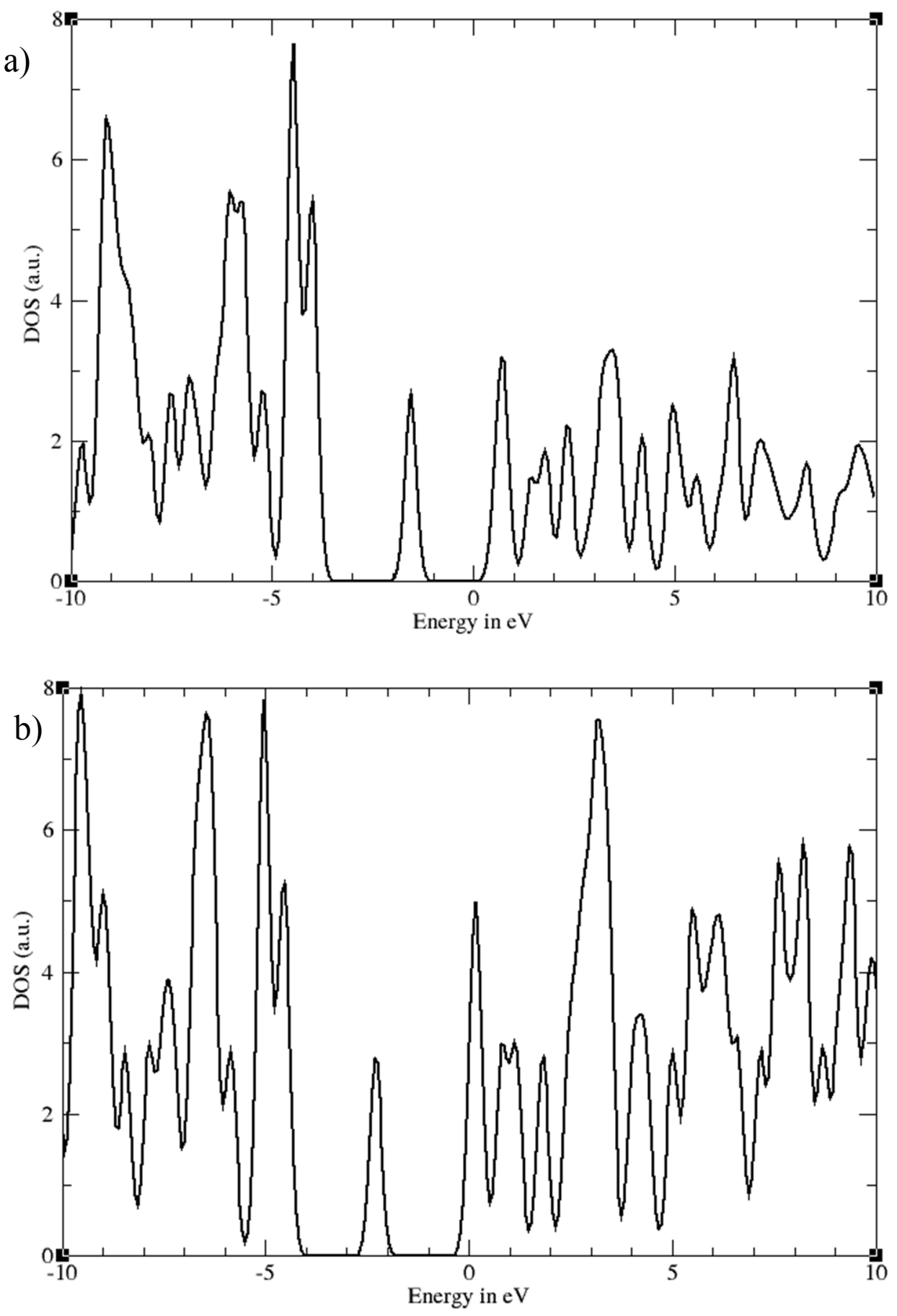

Figure 5: a) CPT DOS for the carboplatin-CNT in the position (3) of Figure 4; b) CPT DOS in gas phase. 
We next expand our research to include a second carboplatin drug to study the ability of the CNT to confine and stabilize CPT molecules. To do it, the first CPT was placed at the center of the CNT nanocarrier and the second CPT molecule was translated progressively inside the CNT from $d=-21 \AA$ and $d=21 \AA$, as for the first CPT molecule. The encapsulation goes through relaxation and electronic structure calculation of the whole system and the adsorption energy for each distance d (Figure 6).

The relaxation process forces the encapsulated second molecule to reposition itself near the CNT centre. As for the first molecule, the same potential barriers shapes are observed near the CNT entrance (positions (1)-(2) on Figure 6). Then, we can observe the formation of two profound adsorption energy valleys inside the CNT. Both present practically the same energy (around $-1.5 \mathrm{eV}$ ) and are located at $-9.8 \AA$ (i.e. $-1.55 \mathrm{eV}$ ) and at $8.2 \AA$ (i.e $-1.46 \mathrm{eV}$ ) from the center of mass respectively. Due to the steric size of the CPT molecule, the central part of the curve shows an increase of the repulsion interaction between CPT and the CPT-CNT system (denoted as (4-1) and (4-2) in Figure 6). All these results show that the second molecule can be also confined inside the CNT. Indeed, in this conformation, it should raise an energy barrier to exit from the nanocarrier around $1.5 \mathrm{eV}$ with no chemical adsorption or bonding occurring between the second molecule and the CPT-CNT system. This can be still confirmed by the projected DOS and the Bader charges analyses for the second molecule in this confined situation that show no modification compared to the gas phase situation (data not shown here).

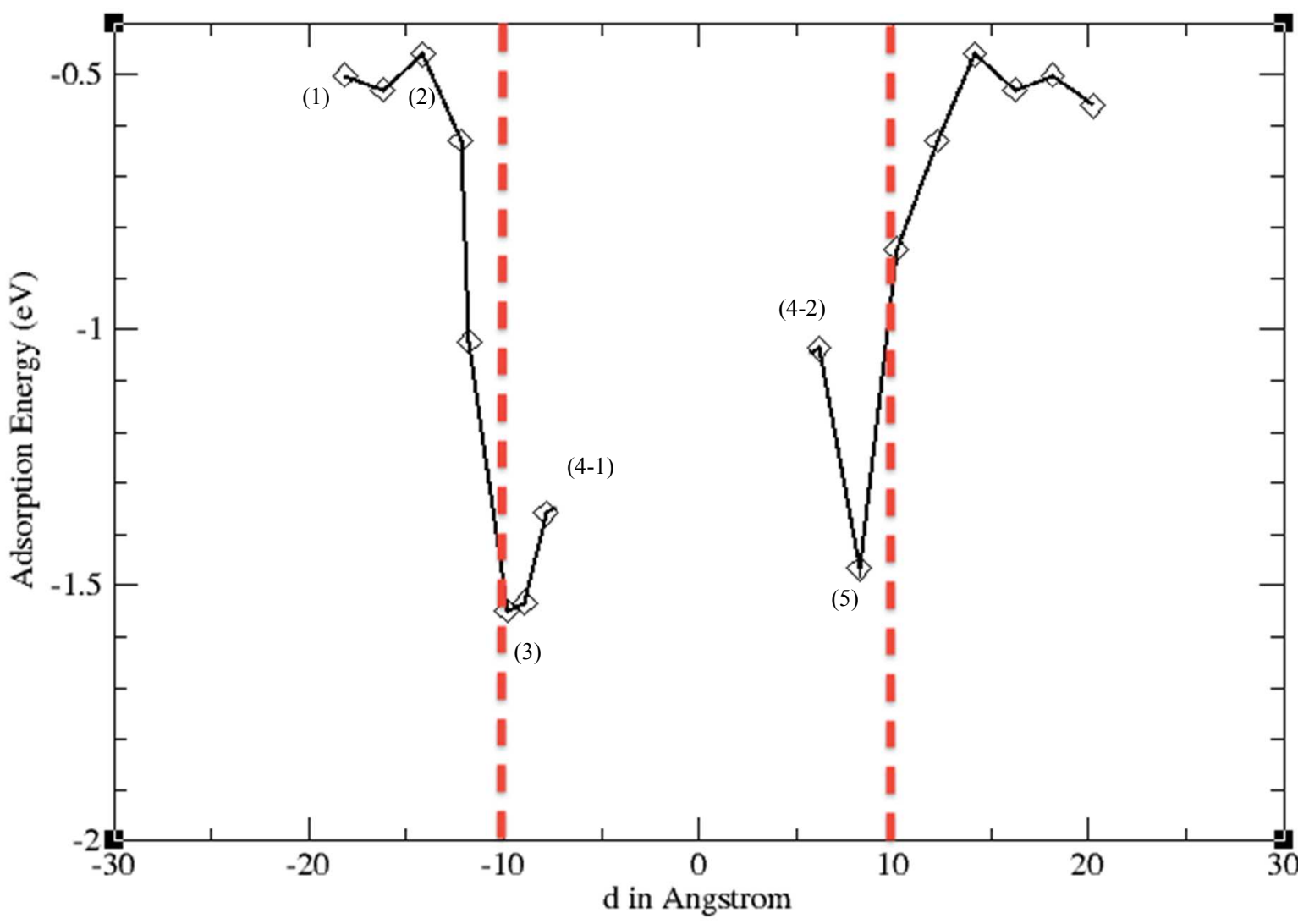


Figure 6: interaction energy of the second CPT as a function of d. Red bars highlight the CNT carbon edges.

\section{Conclusion}

In this study, we show that carboplatin can find stable geometry onto $(10,10)$ CNT sidewalls, but with a rather high energy ($0.13 \mathrm{eV}$ ) compared to the confined situation where the energy is drastically lowered (i.e. $-0.55 \mathrm{eV}$ ). The incorporation of a second therapeutic agent favors the confined state resulting in energy threefold increased (i.e. around $-1.5 \mathrm{eV}$ ). Moreover, when we incorporated a third molecule to completely fill the CNT by the CPT molecules, we find an adsorption energy still lowered and equal to $-2.52 \mathrm{eV}$. Hence, we could hypothesize that each supplementary molecule accounts for around $-1 \mathrm{eV}$ in the adsorption energy when it is highly confined. This leads to a simple empirical law that could describe our results as Eads $=-0.55-(\mathrm{n}-1) \mathrm{eV}$, with $\mathrm{n}$ representing the CPT encapsulated molecule. These results can be compared with the microscopic studies of the carboplatin-filled CNTs carried out by Hampel et al. ${ }^{31,32}$ Indeed, experimental results revealed that carboplatin clusters were incorporated into the opened CNTs whereas occasionally, CPT were found on the outer surface.

Incorporated CPT molecules into CNTs represent a better alternative for drug loading and permits to minimize premature deactivation of CPT drugs before reaching their intended target sites. Moreover encapsulating drugs within the interior cavity of CNTs eliminates any need of forming chemical bonds between drug molecules and CNTs, meanwhile also freeing up the external surface area of CNTs for further functionalization with targeting molecule to increase the selectivity. Indeed, comparison of the density of states for the CPT molecule in gas phase, with the density of states of CPT incorporated inside CPT show slight discrepancies only.

Thanks to Bader's charge analysis, we can verify that the Pt atom of CPT after encapsulation still existed at an identical valence state ( $\mathrm{Pt}$ charge variation between -0.17 e and +0.16 e) regardless of the position of the CPT molecule or their number. We can assume that CPT was in its intact state (i.e. Pt2+) without being oxidized or reduced during physisorption procedure on the inner or outer CNT surface as reported in the literature ${ }^{31,32}$. The important stable state inside the CNT raises the question of the drug release at the appropriated target site. Molecular dynamics, simulating a complete biological system can be the good package to study the experimental release of CPT from the CNT. However, it was shown experimentally that the release of CPT from CNTs, tested by incubation of CNT-CPT in cell-culture medium on many days, needed around 14 days to liberate at least $70 \%$ of the total encapsulated active molecules near the cancer cell. This also corroborates the work performed by Panczyck et al. ${ }^{46}$ who found on a large CNT that the release of cisplatin necessitated to an energy barrier of $25 \mathrm{kcal} / \mathrm{mol}(1.08 \mathrm{eV})$. While extremely fast (below few minutes), experiments developed by Li et al. ${ }^{46}$ on CNT (or Tripisciano et al. on MWCNT) did not reach such velocity to release drug molecules ${ }^{47}$. 
The stability of the drug molecule inside the nanocarrier is thus very strong. In our specific case, one molecule tends to fall into a profound energy well (around $-0.55 \mathrm{eV}$ ), which increases proportionally to the number of supplementary CPT molecules charged into the CNT. It should be noted however, that several parameters (polarization, temperature, etc) could be at the origin of the large discrepancy observed between the theoretical and the experimental observations and that the potential barrier calculated show a large spatial extension which probably hinders the drug release.

\section{Acknowledgments}

Calculations were performed with the supercomputer regional facility Mesocenter of the University of Franche-Comté.

\section{Notes and References}

\footnotetext{
${ }^{a}$ Laboratoire de Nanomédecine, Imagerie et Thérapeutique, EA 4662, Université Franche-Comté (UFR Sciences et Techniques), Centre Hospitalier Universitaire de Besançon, 16 route de Gray, 25030 Besançon, France

${ }^{\mathrm{b}}$ Institut FEMTO-ST, 32 Avenue de l'Observatoire, 25044 Besançon, France

Corresponding Author: fabien.picaud@univ-fcomte.fr
}

1. Yang X. et al., cRGD-functionalized, Biomaterials, 2011, 32, 4151.

2. C. Samori et al., Chem. Comm., 2010, 46, 1494.

3. T. Allen and P. Cullis, Science, 2004, 303, 1818.

4. M. Ferrari, Nat Rev Cancer, 2005, 5, 161.

5. K.K. Jain, Clin Chem, 2007, 53, 2002.

6. P.S. Kim, S. Djazayeri and R. Zeineldin, Gynecol Oncol., 2010, 120, 393.

7. K. Kostarelos, A. Bianco and M. Prato, Nat. Nanotech., 2009, 4, 627.

8. R. Bawa, Nanomedicine, 2009, 5, 5.

9. C. A. Lipinski, F. Lombardo, B.W. Dominy and P. J. Feeney, Adv. Drug Delivery Rev., 2001, 46, 3.

10. M. Prato, K. Kostarelos and A. Bianco, Acc Chem Res., 2008, 41, 60.

11. Z. Liu et al., Nano Res., 2009, 2, 85 . 
12. C. L. Lay, J. Liu, and Y. Liu, Expert Rev. Med. Devices, 2011, 8, 561.

13. A. Bianco, K. Kostarelos and M. Prato, Curr. Opin. Chem. Biol., 2005, 9, 674.

14. J. Y. Chen, S. Y. Chen, X. R. Zhao, L.V. Kuznetsova, S. S. Wong, and I. Ojima, J. Am. Chem. Soc., 2008, 130, 16778.

15. L. Meng, X. Zhang, Q. Lu, Z. Fei and P. J. Dyson, Biomaterials, 2012, 33, 1689.

16. C.L. Lay, J. Liu and Y. Liu, Expert Rev. Med. Devices, $2011,8,561$.

17. H. Ali-Boucetta, K.T. Al-Jamal, D. McCarthy, M. Prato, A. Bianco and K. Kostarelos, Chem. Commun., 2008, 4, 459.

18. S. Dhar, W.L. Daniel, D.A. Giljohann, C.A. Mirkin and S.J. Lippard, J. Am. Chem. Soc., 2009, 131, 14652.

19. S. Dhar, Z. Liu, J. Thomale, H. Dai, S. J. Lippard, J. Am. Chem. Soc., 2008, 130, 11467.

20 S.-T. Yang, X. Wang, G. Jia, Y. Gu, T. Wang, H. Nie, C. Ge, H. Wang, and Y. Liu, Toxicol. Lett., 2008, 181, 182.

21 Z. Liu, C. Davis, W. Cai, L. He, X. Chen and H. Dai, Proc. Natl. Acad. Sci., 2008, 105, 1410.

22 M. L. Schipper, N. Nakayama-Ratchford, C. R. Davis, N. W. S. Kam, P. Chu, Z. Liu, X. Sun, H. Dai, and S. S. Gambhir, Nat. Nanotechnol., 2008, 3, 216.

23 H. Gao, Y. Kong, D. Cui and C. S. Ozkan, Nano Lett., 2003, 3, 471-473.

24 S. Y. Hong et al. Nature Mater. 2010, 9, 485.

25 A. Ilie, J. S. Bendall, K. Nagaoka, S. Egger, T. Nakayama and S. Crampin, ACS Nano, 2011, 5, 2559.

26 M. R. Pederson and J. Q. Broughton, Phys. Rev. Lett., 1992, 69, 2689.

27 T. A. Hilder and J. M. Hill, Small, 2009, 5, 300.

28 N. Tagmatarchis and M. Prato, Pure Appl. Chem., 2005, 77, 1675.

29 S. Kraszewski, E. Duverger, C. Ramseyer and F. Picaud, J. Chem. Phys., 2013, 139, 174704.

30 P. Ordejon, E. Artacho, and J. M. Soler, Phys. Rev. B, 1996, 53, R10441.

31 S. Hampel et al., Nanomedicine, 2008, 3, 175.

32 M. Arlt et al., Nanotechnology, 2010, 21, 335101.

33 A. F. Avila and G. S. R. Lacerda, Mater. Res., 2008, 11, 325. 
34 S. Rols et al., Eur. Phys. J. B, 2000, 18, 201.

35 D. Lu, Y. Li, S. V. Rotkin, U. Ravaioli, and K. Schulten, Nano Lett., 2004, 4, 2383.

36 D. Lu, Y. Li, U. Ravaioli, and K. Schulten, J. Phys. Chem. B, 2005, 109, 11461.

37 P. Hohenberg and W. Kohn, Phys. Rev., 1964, 136, B864.

38 W. Kohn and L.J. Sham, Phys. Rev., 1965, 140, A1133.

39 J.P. Perdew, K. Burke and M. Ernzerhof, Phys. Rev. Lett., 1996, 77, 3865.

40 R. Bader, Atoms in Molecules: A Quantum Theory, Oxford, University Press, New York, 1990.

41 W. Tang, E. Sanville and G. Henkelman, J. Phys.: Cond. Matter, 2009, 21, 084204.

42 E. Sanville, S.D. Kenny, R. Smith and G. Henkelman, J. Comp. Chem., 2007, 28, 899.

43 G. Henkelman, A. Arnaldsson and H. Jónsson, Comput. Mater. Sci., 2006, 36, 254.

44 N.J. Wheate et al., Dalton Transactions, 2010, 39, 8113.

45 L. Kelland, Nat Rev. Cancer, 2007, 7, 573.

46. T. Panczyk, A. Jagusiak, G. Pastorin, W. H. Ang, and J. Narkiewicz-Michalek, J. Phys. Chem. C, 2013, 117, 17327.

47 C. Tripisciano, K. Kraemer, A. Taylor and E. Borowiak-Palen, Chem. Phys. Lett., 2009, 478, 200. 
1

2

3

4

5

6

7

8

9

10

11

12

13

14

15

16

17

18

19

20

21

22

23

24

25

26

27

28

29

30

31

32

33

34

35

36

37

38

39

40

41

42

43

44

45

46

47

48

49

50

51

52

53

54

55

56

57

58

59

60

The table of contents:

The encapsulation of several anti-cancer drugs is energetically favored inside small carbon nanotubes.
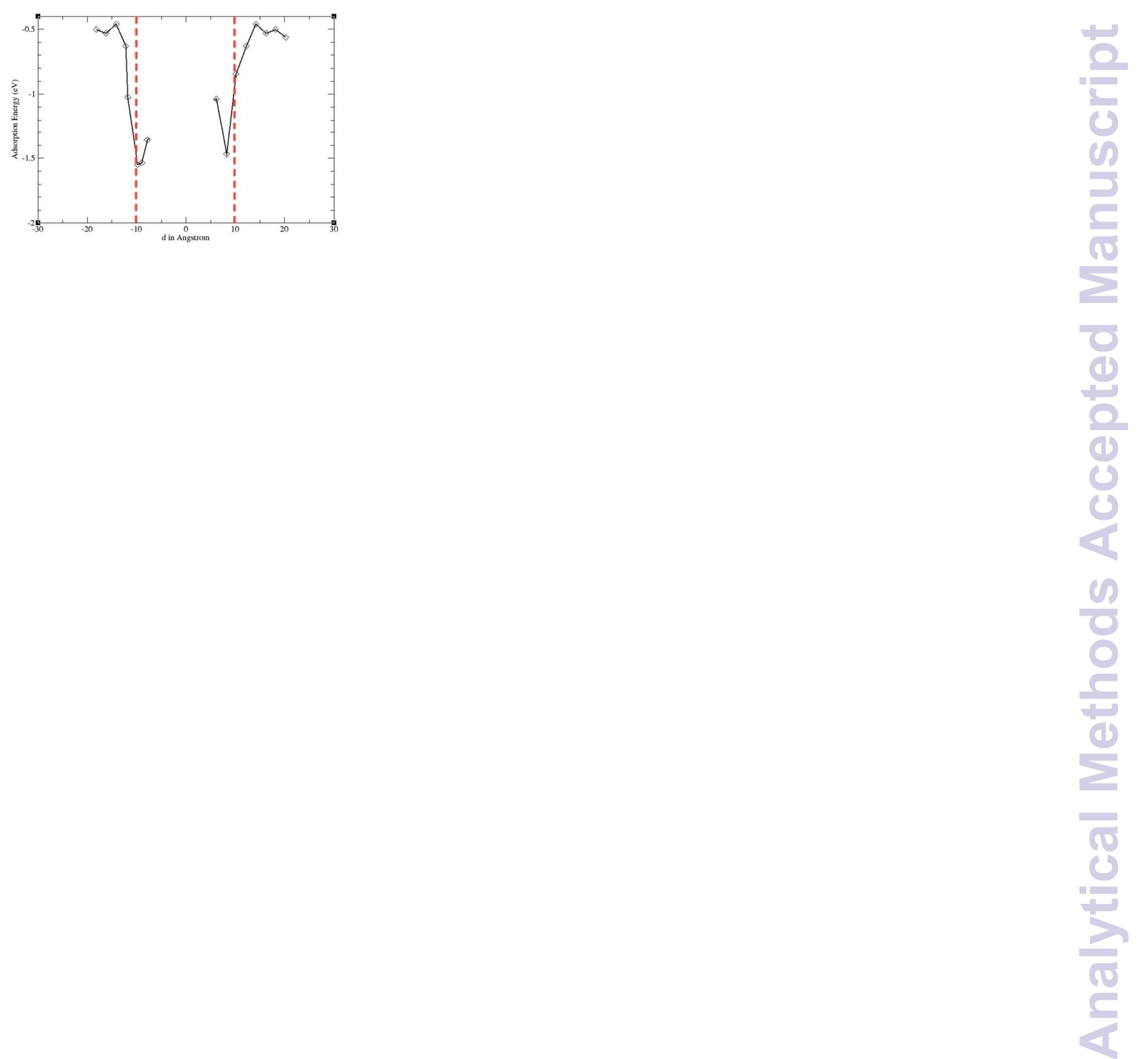